Rev. salud pública. 14 sup (2): 58-68, 2012

\title{
Desplazamiento forzado, niñez y adolescencia: escenarios en relación con su estabilización socioeconómica
}

\section{Forced displacement, childhood and adolescence: scenarios regarding socioeconomic stability}

\author{
Gonzalo A. Patiño y Oscar F. Herrán ${ }^{2}$ \\ 1 Escuela de Economía y Administración, Universidad Industrial de Santander.Bucaramanga, \\ Colombia.gpatiño@uis.edu.co \\ 2 Escuela de Nutrición y Dietética, Universidad Industrial de Santander. Bucaramanga, Colombia. \\ oscar.herran@gmail.com
}

Recibido 25 Mayo 2011/Enviado para Modificación 8 Agosto 2011/Aceptado 3 Noviembre 2011

\section{RESUMEN}

Objetivo Describir y analizar escenarios de estabilización socioeconómica para población en situación de desplazamiento forzado, en los que se preste especial atención a la situación de niños, niñas y adolescentes.

Métodos Se utiliza, a partir de talleres de expertos, el método Analytic Hierarchy Process (AHP), elaborado por el profesor Thomas Saaty con participación investigadores, ONG y de comunidades en situación de desplazamiento del Área Metropolitana de Bucaramanga.

Resultados El resultado parcial de esta investigación muestra como algunos adultos mayores de comunidades en situación de desplazamiento forzado prefieren adaptarse a los centros urbanos, antes que retornar sus lugares de origen, en particular cuando se trata de la protección y el bienestar de sus hijos menores. El índice de consistencia y la relación de consistencia del método están dentro de los parámetros previstos.

Conclusión Es necesario repensar los programas de estabilización socioeconómica promovidos por el gobierno en relación con la población en situación de desplazamiento forzado, en particular cuando se prevén situaciones que comprometen el bienestar de los niños, niñas y adolecentes.

Palabras Clave: Violencia, migración interna, evaluación de necesidades, aceptación de la atención en salud. (fuente: DeCS, BIREME).

\section{ABSTRACT}

Objective Describing and analysing socio-economic stabilisation scenarios for populations which have been forcibly displaced paying special attention to the situation of children and adolescents. 
Method The analytic hierarchy process (AHP) multi-criteria decision-making method developed by Professor Thomas Saaty was used through expert workshops, involving researchers, leaders of NGOs and displaced communities living in the city of Bucaramanga.

Results The partial result of this research showed that some elderly adults from forcibly displaced communities preferred to adapt themselves to urban centres, rather than return to their places of origin, particularly when it came to protecting younger children and dealing with their welfare. The method's consistency index and consistency ratio came within the expected parameters.

Conclusion Government-promoted social and economic stabilisation programmes must be rethought regarding forcibly displaced populations, particularly when situations are envisaged involving the welfare of children and adolescents.

Key Words: Humanitarian Studies Institute (HSI), forced displacement, early recovery, children and adolescents, socio-economic stabilisation scenarios (source: $\mathrm{MeSH}, \mathrm{NLM}$ ).

$\mathrm{E}$ tema de las vulnerabilidades sociales de niños, niñas y adolescentes en situación de desplazamiento y sus posibles alternativas de solución integral no puede escapar a las distintas lógicas de intervención del gobierno, organizaciones y actores institucionales, que con sus esfuerzos propenden por una adecuada estabilización socioeconómica. Se trata, en esencia, de libertades y derechos fundamentales, que se violan con frecuencia (1-4). La estabilización socioeconómica, por su parte, debe comprenderse en un marco amplio de acciones que en el tiempo se desarrollan bajo el amparo del Estado, y que persiguen mejorar las condiciones de vida de quienes se han visto obligados a abandonar sus lugares de residencia habitual.

Los factores de mayor peso son los que se relacionan con el conflicto armado interno y que afectan notablemente el sentido de la justicia social, así como la institucionalidad (5-7). La Ley 387 de julio 18 de 1997 señala en sus artículos 16-18 los planes y programas que deben permitir el acceso a la oferta institucional disponible, así como el momento en que cesa la condición de desplazado forzado. Esta última involucra directamente a las personas afectadas, en términos de la cooperación que debe existir para que el retorno a los lugares de origen o su reasentamiento sean una realidad.

En este sentido es preciso aclarar, que la estabilización de los jefes de hogares en situación de desplazamiento es vital para evitar que los menores de estas familias se incorporen activamente al mercado laboral sea 
el informal o no. El trabajo infantil viola las elementales libertades del ser humano y se ha pretendido corregir en diversas cartas y acuerdos, aunque no se constituye precisamente en una sorpresa, pues está fuertemente correlacionada con el nivel promedio del ingreso en una sociedad (8-9). Se ha constatado ampliamente, que el ingreso nacional per cápita está fuertemente relacionado con el contexto en el que se ubican y desarrollan las actividades de las familias (10).

En nuestro medio con frecuencia se muestran los balances del mercado laboral en las principales áreas metropolitanas, sin medirse el impacto causado por la flexibilización del aparto productivo en materia de pobreza y vulnerabilidad. Especialmente se carece de una información detallada en relación con los altos niveles de subempleo e informalidad, al permitirse el acceso al trabajo en condiciones de precariedad de niños, niñas y adolescentes, corriéndose el riesgo adicional de que abandonen sus estudios y remplacen a los adultos desempleados (11).

Como se sabe la población en situación de desplazamiento forzado presenta mayores grados de riesgo y vulnerabilidad (12-13); su inclusión al mercado laboral se da en condiciones que algunos autores señalan como "inclusión desigual" (14). En nuestro caso ello se debe a que estos mismos grupos han sido ya excluidos cuando, por ejemplo, se les ha violado sus derechos humanos y libertades fundamentales (amenaza, tortura, secuestro $\mathrm{y}$ asesinato). Es decir, se les vincula a los subsistemas funcionales como el de salud, educación, vivienda y generación de ingresos en condiciones que terminan reforzando las "desigualdades estructurales" existentes en la sociedad y abriéndose paso un nuevo conjunto de "desigualdades dinámicas" (15). Lo notable de esta situación es que para lograr mayores niveles de equidad la Corte Constitucional ha procedido a declarar un estado de cosas inconstitucionales, solicitando se verifique el cumplimiento de la política pública en materia de derechos. Con ello podemos afirmar, que las inequidades que usualmente se identifican en relación con esta población, y en particular los niños, niñas y adolescentes son de diversa naturaleza y origen.

Uno de los aspectos a destacar es la alta participación (55,1\%), de niños, niñas y adolecentes en el total de la población desplazada inscrita en el RUPD, así como la no inscrita $(51,6)$, que tiene menos de 18 años de edad, frente a un $37 \%$, que representa la población total censada en ese mismo rango de edad 
según los últimos censos. Esta condición demográfica afecta sensiblemente sus derechos tanto en el corto como en el mediano plazo y genera una espiral negativa que se extiende a otros ámbitos en los que su participación se ve seriamente comprometida, en particular en relación a sus posibilidades de acceso a la educación, a la salud y al sistema de atención a la primera infancia (16).

Uno de los dramas más comunes que acompañan el desarrollo individual de los niños y su proyección en la vida social en la modernidad es el paulatino y temprano desarraigo al que someten en los centros urbanos cuando los adultossus padres o familiares-deben engancharse rápidamente al mercado laboral y responder a los avatares y exigencias propias del trabajo productivo y eficaz, que exige la sociedad opulenta y esnobista de nuestra época (17-18). Este "sacrificio", se realiza en virtud de un pretendido bienestar, que se supone debe extenderse a los menores de edad.

En el caso de los niños, niñas y adolescentes desplazados, el problema es de mayor complejidad, pues el anclaje de sus padres y familiares al sistema productivo urbano suele producirse en condiciones de mayor precariedad, dado su escaso capital social, nivel de formación y limitadas posibilidades de vincularse mediante un contrato legal.

Un alto porcentaje de los menores que componen estos hogares $(8,3 \%)$, no vive con ninguno de sus padres y el 34,7 \%, adicional vive con uno sólo de los progenitores, en su altísima mayoría con la madre, lo cual los expone a otros tipos de riesgo (16).

\section{METODOLOGIA}

Este trabajo forma parte del proyecto de investigación "Evaluación de Impacto de Estabilización socioeconómica de la población en situación de desplazamiento en el Área Metropolitana de Bucaramanga", que se adelanta con recursos internos de la UIS, en una de las líneas de investigación en temas humanitarios (recuperación temprana), que promueve el Instituto de Estudios Humanitarios (IEH) y forma parte de las actividades, que adelanta el Nodo de este instituto en Bucaramanga.

La metodología consiste en la aplicación del Analytic Hierarchy Process (AHP), que ha sido exitosamente aplicada por el profesor Thomas Saaty (19). 
Para el presente estudio se desarrolla con base en los principios de identidad y descomposición del objetivo general (estabilización socioeconómica a partir de la variables empleo y protección de la niñez), propuesto en relación a tres escenarios (retorno, reubicación en un lugar similar al de origen, adaptación al contexto urbano), el establecimiento de unos criterios o factores que realmente incidan en el objetivo y la formulación de síntesis de prioridades. El análisis de jerarquías comprendió la realización de cuatro talleres de expertos, que adelantaron en Piedecuesta, Santander; en este caso participaron lideres de población en situación de desplazamiento con el acompañamiento de ONG`s del Área Metropolitana de Bucaramanga (Fundación Estructurar, Visión Mundial) e investigadores.

La solución del problema consiste en un proceso mediante el cual durante varias etapas se establecen prioridades. En la primera etapa se determinan los elementos más importantes del problema o realidad objetivo; en la segunda etapa se trata de establecer el mejor procedimiento de verificación de la observación, ensayo y calificación de los elementos. La tercera etapa consiste en la elaboración de un método de toma de decisiones y de calificación de la calidad del mismo. Todo el proceso se somete a corrección hasta que exista plena seguridad que se abarcaron todas las etapas del mismo adquiriendo un carácter sistémico (20). La jerarquía se considera completa si el elemento de un nivel dado funciona como criterio para todos los elementos de los niveles inferiores.

El problema consiste en la elección de una de estas tres alternativas: Retorno, Reubicación, Adaptación, teniendo como variable principal del estudio lograr niveles de empleo para adultos-a partir de programas de generación de ingresos-, que permitan a los niños, niñas y adolescentes alejarse del empleo informal. En el Método (AHP), los elementos del problema (realidad objetivo), se comparan en forma de pares con respecto a su influencia en una característica (objetivo, criterio, etc.), a partir de una escala de intensidad relativa (Cuadro 1). Se forma, de esta manera, una matriz que se somete a las propiedades algebraicas de la misma (simetricidad inversa entre otras).

En la siguiente etapa, denominada síntesis de prioridades, los expertos ya han construido la matriz y han expresado sus distintas valoraciones. Se determina la intensidad relativa, la magnitud, el valor, la deseabilidad o probabilidad de cada objeto por separado con ayuda de la solución algebraica de las matrices propuestas. Para ello es necesario obtener un conjunto de vectores para cada 
matriz y posteriormente normalizar el resultado. En primera instancia se multiplican los elementos de cada fila y se saca una raíz de grado $\mathrm{n}$ (cantidad de elementos en la fila).

Cuadro 1. Escala de Intensidad Relativa

\begin{tabular}{|c|c|c|}
\hline Importancia & Definición & Explicación \\
\hline 1 & De igual importancia & $\begin{array}{l}\text { Igual contribución de dos tipos de } \\
\text { actividades al objetivo general. }\end{array}$ \\
\hline 3 & $\begin{array}{l}\text { Superioridad moderada de un } \\
\text { criterio sobre otro }\end{array}$ & $\begin{array}{c}\text { La experiencia de una ligera } \\
\text { superioridad con respecto a otro. }\end{array}$ \\
\hline 5 & Fuerte Superioridad & La experiencia habla de ello \\
\hline 7 & Importancia Significativa & $\begin{array}{c}\text { A un tipo de actividad se le da } \\
\text { tal importancia que se vuelve } \\
\text { significativo. }\end{array}$ \\
\hline 9 & Superioridad Indiscutible & Se admite sin discusión. \\
\hline 2 & $\begin{array}{l}\text { Decisiones Intermedias entre dos } \\
\text { apreciaciones cercanas }\end{array}$ & $\begin{array}{l}\text { Se utiliza en casos de convenio y } \\
\text { compromiso. }\end{array}$ \\
\hline 4 & $\begin{array}{l}\text { Decisiones Intermedias entre dos } \\
\text { apreciaciones cercanas }\end{array}$ & $\begin{array}{l}\text { Se utiliza en casos de convenio y } \\
\text { compromiso. }\end{array}$ \\
\hline 6 & $\begin{array}{l}\text { Decisiones Intermedias entre dos } \\
\text { apreciaciones cercanas }\end{array}$ & $\begin{array}{l}\text { Se utiliza en casos de convenio y } \\
\text { compromiso. }\end{array}$ \\
\hline 8 & $\begin{array}{l}\text { Decisiones Intermedias entre dos } \\
\text { apreciaciones cercanas }\end{array}$ & $\begin{array}{l}\text { Se utiliza en casos de convenio y } \\
\text { compromiso. }\end{array}$ \\
\hline Magnitudes Inversas & $1 / 2,1 / 3,1 / 4,1 / 5 \ldots 1 / 9$ & \\
\hline
\end{tabular}

Cada uno de los coeficientes obtenidos se divide por con la sumatoria total de estos coeficientes, obteniendo el respectivo vector de coeficientes $\mathrm{x}$.

Componente del vector propio de la primera fila:

$$
\sqrt[4]{\frac{W 1}{W 1} \times \frac{W 1}{W 2} \times \frac{W 1}{W 3} \times \frac{W 1}{W 4}}=a
$$

Componente del vector propio de la segunda fila:

$$
\sqrt[4]{\frac{W_{2}}{W_{1}} \times \frac{W_{2}}{W_{2}} \times \frac{W_{2}}{W_{3}} \times \frac{W_{2}}{W_{4}}}=b
$$

Componente del vector propio de la tercera fila:

$$
\sqrt[4]{\frac{W_{3}}{W_{1}} \times \frac{W_{3}}{W_{2}} \times \frac{W_{3}}{W_{3}} \times \frac{W_{3}}{W_{4}}}=c
$$

Normalizar el resultado para obtener la calificación del vector de prioridades

$$
\Sigma=a+b+c+d ; \frac{a}{\Sigma}=X_{1} ; \frac{b}{\Sigma}=X_{2} ; \frac{c}{\Sigma}=X_{3} ; \frac{d}{\Sigma}=X_{3}
$$


Cuando la matriz tiene esta forma en realidad $X_{1}, X_{2}, X_{3}, X_{4}$, no es más que $W_{1}, W_{2}, W_{3}, W_{4}$. De la relación $W_{i} / W j$ determinamos cada componente. Es necesario añadir que en la matriz de conceptos (opiniones) no hay relaciones de tipo $\mathrm{Wi} / \mathrm{Wj}$, solamente se tienen números enteros o magnitudes inversas de la escala.

$$
\left(\begin{array}{llll}
\frac{W_{1}}{W_{2}} & \frac{W_{1}}{W_{2}} & \frac{W_{1}}{W_{3}} & \frac{W_{1}}{W_{4}} \\
\frac{W_{2}}{W_{1}} & \frac{W_{2}}{W_{2}} & \frac{W_{2}}{W_{3}} & \frac{W_{2}}{W_{4}} \\
\frac{W_{3}}{W_{1}} & \frac{W_{3}}{W_{2}} & \frac{W_{3}}{W_{3}} & \frac{W_{3}}{W_{4}} \\
\frac{W_{4}}{W_{1}} & \frac{W_{4}}{W_{2}} & \frac{W_{4}}{W_{4}}
\end{array}\right) *\left[\begin{array}{c}
X_{1} \\
X_{2} \\
X_{3} \\
X_{4}
\end{array}\right]=\left[\begin{array}{c}
Y_{1} \\
Y_{2} \\
Y_{3} \\
Y_{4}
\end{array}\right]
$$

El problema algebraico de consistencia plantea la solución de una ecuación

$$
\begin{gathered}
\text { tipo: } \mathrm{AW}=\mathrm{nW}, \\
A=\left(\frac{W i}{W j}\right)
\end{gathered}
$$

El problema general de juicios inversamente simétricos consiste en la solución de la ecuación: $A^{\prime} W^{\prime}=\lambda_{\max } W$; donde $\lambda$ máx es el significado más alto de la matriz de conceptos $A$. Acto seguido se "normaliza" el resultado con el propósito de obtener un vector de prioridades. En un comienzo se suma cada columna de juicios (conceptos), después la suma de la primera columna se multiplica por la magnitud del primer componente del vector normalizado de prioridades; la suma de la segunda columna por el segundo componente $y$, así sucesivamente. La suma así obtenida nos da $\lambda$ max.

Posteriormente se calcula un índice de consistencia que nos da información acerca del grado de obstrucción cardinal y transitiva de las comparaciones pares. Para este índice tenemos Icon $=\left(\lambda_{\max }-n\right) /(n-1)$, donde n- número de elementos comparados. Para una matriz inversamente simétrica siempre se cumple la siguiente condición: $\lambda \geq n$. Acto seguido comparamos esta magnitud con aquella se obtendría de una elección casual de juicios cualitativos de una escala $1 / 9,1 / 8,1 / 7, \ldots, 1,2, \ldots 9$. A continuación se presentan "medias "de concertación para matrices de diferente nivel (Tabla 1). 
Tabla 1. Medias Aritméticas de Consistencia para Matrices

\begin{tabular}{lcccccccccc}
\hline $\begin{array}{l}\text { Medida de la matriz } \\
\text { Relación de }\end{array}$ & 1 & 2 & 3 & 4 & 5 & 6 & 7 & 8 & 9 & 10 \\
consistencia & 0 & 0 & 0,58 & 0,9 & 1,12 & 1,24 & 1,32 & 1,41 & 1,45 & 1,49 \\
\hline
\end{tabular}

Si dividimos el Índice de consistencia (Icon) sobre el número que corresponde a la relación de consistencia para una matriz de orden $n$ obtendremos el índice relación de consistencia (Irel). Esta magnitud deberá ser de un $10 \%$, o menos para ser permisible y, en ningún caso superior al $20 \%$. Si el índice sale de estos límites, entonces los expertos deben probar de nuevo los juicios establecidos.

\section{RESULTADOS}

El resultado del taller realizado con participación de expertos es el que a continuación se presenta (Cuadro 2). Se toma como punto de partida la siguiente pregunta. A partir de de la realidad objetivo (estabilización socioeconómica) y teniendo en cuenta un conjunto de variables (recursos financieros, voluntad política, recurso humano y presencia institucional), claves para el bienestar de los niños, niñas y adolescentes ¿Cuál de los tres escenarios alternativos-retorno, reubicación en un lugar similar al anterior, adaptación a la ciudad-, es posible en el tiempo y por qué?

\begin{tabular}{lccccccc}
\multicolumn{8}{c}{$\begin{array}{c}\text { Cuadro 2. Construcción de escenarios alternativos teniendo en } \\
\text { consideración la estabilidad de niños, niñas y adolescentes }\end{array}$} \\
$\begin{array}{lllllll}\text { Niños, niñas y } \\
\text { adolescentes }\end{array}$ & $\begin{array}{c}\text { a: } \\
\text { Retorno }\end{array}$ & $\begin{array}{c}\text { b: } \\
\text { Reubicación }\end{array}$ & $\begin{array}{c}\text { c: } \\
\text { Adaptación }\end{array}$ & MULTIPLIC & $\begin{array}{c}\text { Coefic. } \\
\text { a...c }\end{array}$ & $\begin{array}{c}\text { Coefic. } \\
\text { X }\end{array}$ & $\lambda$ max \\
\hline $\begin{array}{l}\text { a: Retorno } \\
\text { b: Reubicación }\end{array}$ & 1 & $1 / 3$ & $1 / 5$ & 0,067 & 0,39 & 0,110 & 1,21 \\
c: Adaptación & 7 & 1 & 1 & 3 & 1,44 & 0,407 & 0,94 \\
Totales & 11 & 1 & 1 & 5 & 1,71 & 0,483 & 1,06 \\
\hline
\end{tabular}

La adaptación, comparada con las otras dos alternativas (retorno y reubicación en un lugar similar al que vivían), se consideró como de mayor posibilidad. En el cuadro de construcción de alternativas se cumplieron las restricciones paramétricas $(\lambda \max \geq 3,21)$, así como los índices de consistencia y relación de consistencia (Icon=0, 105; Irel=18 \%). La relación de consistencia exigida por la metodología de análisis concuerda con los límites implícitamente establecidos para ella (10\% o menos); Es preciso aclarar, que se asume para esta etapa la posibilidad de un rango 
mayor, lo que no implica pérdida de coherencia interna en la discusión a lo largo de todo el proceso y al mismo tiempo establecer un margen de error aceptable para la metodología en cuestión.

Una vez instalados en la ciudad e inscritos en un sistema que permite, aunque de manera precaria, acceso a seguridad social, servicios públicos domiciliarios y generación de ingresos, la adaptación al contexto urbano se presenta como una mejor opción, frente a aquella que tenían en las zonas rurales; Estas comunidades son conscientes, sin embargo, que se requieren políticas de empleo y educación, que les permita a ellos y a sus hijos mejores condiciones de vida y superar el estado de cosas inconstitucional declarado por la Corte. Si esto se cumple, las personas en situación de desplazamiento no querrán volver con sus hijos al sitio de origen por el temor a las masacres, los asesinatos selectivos, las desapariciones forzadas, el secuestro, el reclutamiento forzado al que pueden someterse los menores de edad. Los conflictos armados producen no solo pérdida de bienes materiales, muertes, heridas y discapacidades físicas, ellos dejan huellas profundas en las vidas de las personas, las familias y la sociedad.

\section{DISCUSION}

El proyecto de investigación aborda una perspectiva particular derivada del conflicto armado en Colombia, a saber: la perentoria necesidad que tiene el Estado colombiano y la sociedad, en su conjunto, de abordar de manera radical y de carácter urgente el tema del despoblamiento que ha provocado la guerra en los territorios del país, con consecuencias alarmantes para la institucionalidad en su conjunto (21). En ese sentido, el análisis de los programas de estabilización socioeconómica, que involucra especialmente las situaciones de niños, niñas y adolecentes, debe fundarse en un criterio más amplio y superar el criterio técnico, que prevalece en la evaluación de los programas del Gobierno (22). Por el contrario, se trata de una mirada a la complejidad del ordenamiento social producida por quienes hoy habitamos este espacio y del carácter éticopolítico que como ciudadanos debemos emprender para restituir en parte el daño causado a la sostenibilidad del territorio y a quienes en él habitan (23).

No podemos seguir habitando nuestras ciudades e ignorando la miseria, el hambre y la desnutrición, que padecen los menores de edad, en particular aquellos, que ya han enfrentado fuertes fenómenos de exclusión social motivados por el conflicto armado (24). 
En el caso de los niños, niñas y adolescentes en situación de desplazamiento forzado se trata de personas que han perdido buena parte de su capital social y se someten a un fuerte desarraigo en las ciudades, lo cual requiere un mayor periodo de adaptación a las condiciones urbanas, de lo contrario su caso podría verse como de "adaptación forzada". En otras palabras, rompen violentamente con su hábitat y se instalan precariamente en otro que los acoge (25).

El retorno puede significar, para los menores de edad y sus familias, instalarse de nuevo en un entorno asociado a los cultivos ilícitos, al reclutamiento forzado, sistemático y progresivo, a campos minados, y al tráfico de armas. Un lugar, en fin, en el que la autoridad y la justicia la ejercen con frecuencia actores armados ilegales.

El tema de la estabilización socioeconómica para estos grupos poblacionales no puede partir de las decisiones del mercado, pues ellas se sustentan en la idea de la libertad económica, que en estos casos no opera de manera eficiente y si puede introducir situaciones de inequidad y mayor injusticia social. Varios caminos quedan por explorar; Primero, le corresponde al Estado superar el Estado de Cosas Inconstitucional (ECI), declarado por la Corte Constitucional. Para ello será necesario consolidar los proyectos de ley que actualmente se discuten (Ley de Tierras y Ley de Víctimas); Segundo, le corresponde a la empresa privada participar decididamente en proyectos que permitan a personas en situación de desplazamiento incorporarse a determinados eslabones del sector productivo y generar oportunidades y capacidades. Tercero, le corresponde a la población en situación de desplazamiento involucrarse en procesos de capacitación, acercarse a la labor de proyección social que realizan organizaciones del Estado, ONG`s y centros universitarios, consolidar una conciencia en el núcleo familiar que favorezca la inclusión de los menores al sistema educativo, y alejarlos de las redes de trabajo informal

\section{REFERENCIAS}

1. Sen A. El derecho a no tener hambre. Colombia. Universidad Externado de Colombia. Traducción de Everaldo lamprea; 2002; p. 15.

2. Sen A. La libertad individual como compromiso social. 2da Edición. Quito, Ediciones Abya - Yala; 1999.

3. Sen A. Economía de bienestar y dos a aproximaciones a los derechos. Bogotá, Universidad externado de Colombia. Centro de investigación en Filosofía y Derecho; 2002.

4. Sen A. Desarrollo y Libertad. Barcelona, Editorial Planeta; 2001. 
5. Rawls J. La justicia como equidad: una reformulación. Barcelona, Editorial Paidós Estado y Sociedad; 2002.

6. Walzer M. Las esferas de la justicia. Primera Edición. México, Fondo de Cultura Económica; 2001.

7. Parijs V, Arnsperger C. Ética económica y social: teorías de la sociedad justa. 1era Edición. Barcelona, Paidós Estado y Sociedad; 2002.

8. Dessy S, Pallage S. Child Labor and Coordination Failures. Journal of Development Economics. 2001; 65(2):469-476.

9. Edmonds E, Pavcnik N. Child Labor in the Global Economy. Journal of Economic Perspectives. 2005; 18(1):199-220.

10. Doepke M, Zilibotti F. The Macroeconomics of Child Labor Regulation. American Economic Review. 2005; 95(5):1492-524.

11. Maecha JC, Salamanca, RB. Evaluación del ajuste y desajuste en niños y jóvenes de estrato socioeconómico bajo de Bogotá. Revista Infancia, Adolescencia y Familia. 2006; 1(2): 341-355.

12. Comisión de Seguimiento a la Política pública sobre el Desplazamiento Forzado. Proceso Nacional de verificación de los Derechos de la población Desplazada. Primer Informe a la Corte Constitucional, Bogotá; 2008.

13. Garay LJ, Barberi G, Prada G, Ramírez C, Cardona I. Verificando el cumplimiento de los derechos. Comisión de seguimiento a la Política Pública sobre desplazamiento forzado. 1era Edición. Bogotá, CODHES; 1998.

14. Sen A, Kilsberg B. Primero la gente. España, Editorial Deusto; 2007, p. 64.

15. Fitoussi JP, Rosanvallon P. La nueva era de las desigualdades. Buenos Aires, Editorial Manantial; 1997.

16. Comisión de Seguimiento a la Política pública sobre el Desplazamiento Forzado. El reto ante la tragedia humanitaria del desplazamiento forzado: Superar la exclusión social de la población desplazada. Tercer Informe a la Corte Constitucional. Bogotá; 2009.

17. Corea C, Lewkowicz, I. ¿Se acabó la Infancia? Ensayo sobre la destitución de la Niñez. Buenos Aires, Editorial Lumen/ Hvmanitas; 1999.

18. Veblen T. Teoria de la clase ociosa. 1era Edición. México, Fondo de Cultura Económica; 2004.

19. Saaty TL. The Analytic Hierarchy Process. New York, McGraw Hill; 1980.

20. Bertalanffy L. The History and Status of General Systems Theory. In: George Lir. Trends in General Systems Theory, New York: Wiley Interscience; 1972.

21. Garay, S, Albarán E, León I, Guerrero B. La Captura y Reconfiguración Cooptada del Estado en Colombia. Bogotá, Fundación Método, Fundación Avina y Transparencia por Colombia; 2008.

22. PNUD, DNP, Acción Social. Hacia una Colombia equitativa e incluyente. Informe de Colombia. Objetivos de desarrollo del Milenio, Bogotá; 2005. p 37.

23. Puntalino S. Uncharted Territory: Land, Conflict and Humanitarian Action. UK, Overseas Development Institute; 2009.

24. Vera AV, Parra F, Parra R. Los Estudiantes Invisibles. Ibagué, Universidad de Ibaqué; 2008.

25. USAID, FUPAD, UDI. Un convenio para el fortalecimiento de las organizaciones de población desplazada en Santander. Informe de ejecución 2009-2010. Bogotá, USAID; 2010; p.17-169. 